\title{
Effects of Crystallization on Dynamic Properties of an Organic Hybrid Consisting of Chlorinated Polyethylene and Hindered Phenol
}

\author{
Chifei $\mathrm{WU}^{\dagger}$ and Saburo AKIYAMA* \\ Institute of Material Science and Technology, East China University of Science \\ and Technology, 130 Meilong-lu, Shanghai 200237, China \\ *Department of Organic and Polymer Materials Chemistry, Tokyo University of Agriculture \\ and Technology, 2-24-16 Naka-cho, Koganei, Tokyo 184-8588, Japan
}

(Received February 15, 2002; Accepted August 21, 2002)

KEY WORDS Dynamic Property / Morphology / Crystallization / Hindered Phenol / Chlorinated
Polyethylene /

Reinforcement of elastomers by the incorporation of inorganic fillers has been the subject of numerous investigations. The additive effects of organic fillers such as polymeric micro-particulates on the mechanical properties of a matrix polymer have also been reported recently. ${ }^{1}$ However, little less attention has been paid to the additive effects of small molecular substance on the performances of elastomers.

In recent studies, ${ }^{2,3}$ our attention has been focused on the discovery of unknown functions of a polymer elicited by the addition of small molecular substances. It was found that the addition of 3,9-bis $\{1,1-$ dimethyl-2[ $\beta$-(3-tert-butyl-4-hydroxy-5-methylphenyl) propionyloxy] ethyl $\}$-2,4,8,10-tetraoxaspiro[5,5]-undecane (AO-80) into chlorinated polyethylene (CPE) caused a novel transition above the glass-transition temperature of CPE. It has also been revealed that $\mathrm{AO}-80$ is a polymorphous substance, i.e., initial AO- 80 that usually crystallized from a dilute solution is a complete crystal, whereas AO- 80 obtained by cooling from its melting state is an amorphism. In addition, the vitrified AO-80 (labeled VAO-80) particles can be crystallized again in CPE matrix by annealing treatment, although the microstructure of this crystal, which includes some CPE chains, is different from that of the initial AO-80. ${ }^{4}$ The AO-80-rich crystalline particle can be regarded as a dynamic multifunctional cross-linking point. Consequently, the crystallized $\mathrm{CPE} / \mathrm{AO}-80$ was found to be a new type of thermoplastic elastomer.

On the other hand, it is well known that rubber filled with either an inorganic or organic filler shows a typical nonlinear viscoelastic behavior: dynamic moduli depend on the strain amplitude and a significant decrease in storage modulus occurs at a strain of about $1 \%$. This effect is generally called the "Payne effect". ${ }^{5,6}$ It has been widely accepted that the Payne effect is related to the filler network formed in the polymer matrix, and it is thought to serve as a measure of the filler network. ${ }^{7}$ The Payne effect is also thought to be of useful for evaluation of a network consisting of AO-80-rich crystalline particles.

In this article, we describe how the dynamic properties of a CPEAO- 80 hybrid over a wide range of strain amplitudes are affected by the incorporation of CPE chains into the AO- 80 crystal. In addition, we propose a new method for estimating network strength. This new approach successfully accounts for the dynamic properties observed by the structure of the annealed CPE/VAO-80 hybrid.

\section{EXPERIMENTAL}

The CPE used as a matrix in this study, with a chlorination degree of $40 \mathrm{wt} \%$, was a rubbery grade (Daisolac RA140, Daiso Co., Japan). The small molecular substance was commercial antioxidant (Asahi Denka Industries Co. ADK STAB AO-80, its chemical structure was shown in Figure 1 of ref 2). The CPE powders were first kneaded with a mixing roller for $5 \mathrm{~min}$. The VAO- 80 particles were then added to the kneaded CPE. The VAO- 80 content was $30 \mathrm{wt} \%$. The mixture was kneaded for $10 \mathrm{~min}$ again at room temperature. Then this kneaded CPE/VAO-80 specimens were placed in a constant temperature chamber at $100{ }^{\circ} \mathrm{C}$ for $4 \mathrm{~h}$ and then quenched into ice-water bath.

The morphologies of the annealed specimens were

${ }^{\dagger}$ To whom correspondence should be addressed (Tel: +86-21-64252569, Fax: +86-21-64252569, E-mail: wucf@ecust.edu.cn). 
C. WU AND S. AKIYAMA

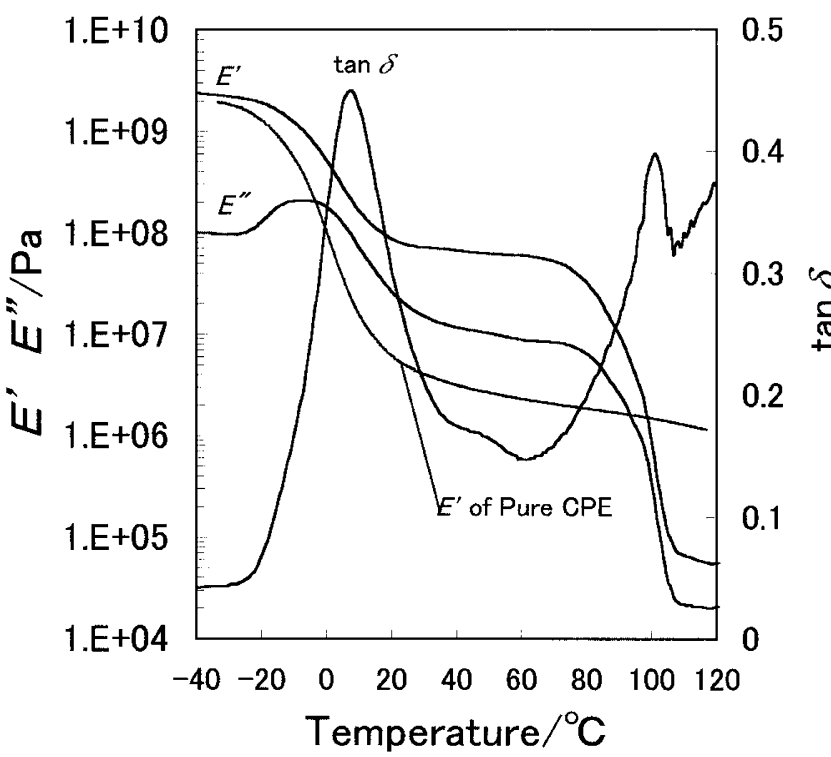

Figure 1. Temperature dependence of storage modulus $E^{\prime}$, loss modulus $E^{\prime \prime}$, and tangent $\tan \delta$ at $11 \mathrm{~Hz}$ for annealed CPE/VAO-80 (30 wt $\%)$.

observed by a scanning electron microscopy (SEM, Hitachi S-4500 field emission gun scanning electron microscope). SEM micrographs of the samples were taken from the fractured surfaces, which were sputtered with gold before viewing.

Dynamic mechanical properties measurements were carried out using a dynamic mechanical analyzer (DVE-V4, Rheology Co., Japan) on sample specimens $10 \mathrm{~mm}$ in length, $5 \mathrm{~mm}$ in width, and about $1 \mathrm{~mm}$ in thickness. The samples were subjected to two types of tests, strain sweeps and temperature sweeps. In the case of strain sweeps, double strain amplitudes (DSA = $(2 \times A / L) \times 100(\%)$, where $A$ is the amplitude of vibration and $L$ is the length of the sample) ranging from 0.03 to $20 \%$ were applied with a frequency of $11 \mathrm{~Hz}$ at a constant temperature of $30^{\circ} \mathrm{C}$. Temperature sweeps measurement was carried out at temperatures ranging from -40 to $120^{\circ} \mathrm{C}$ and at a heating rate of $3{ }^{\circ} \mathrm{C} \mathrm{min}^{-1}$. The test frequency was fixed at $11 \mathrm{~Hz}$, and strain amplitude was set at $1 \%$ DSA.

\section{RESULTS AND DISCUSSION}

Figure 1 shows the temperature dependencies of storage modulus $E^{\prime}$, loss modulus $E^{\prime \prime}$, and tangent $\tan \delta$ for annealed CPE/VAO-80 (30 wt $\%$ ). This sample clearly exhibited two transitions. This is different from untreated CPE/VAO-80 (see Figure 5 of ref 3), in which the second transition appeared at about $50^{\circ} \mathrm{C}$. The first transition is attributed to the glass transition of the CPE matrix phase, whereas the second transition is associated with the melting of the crystallized AO-80 particles because its temperature is the same with the melt-

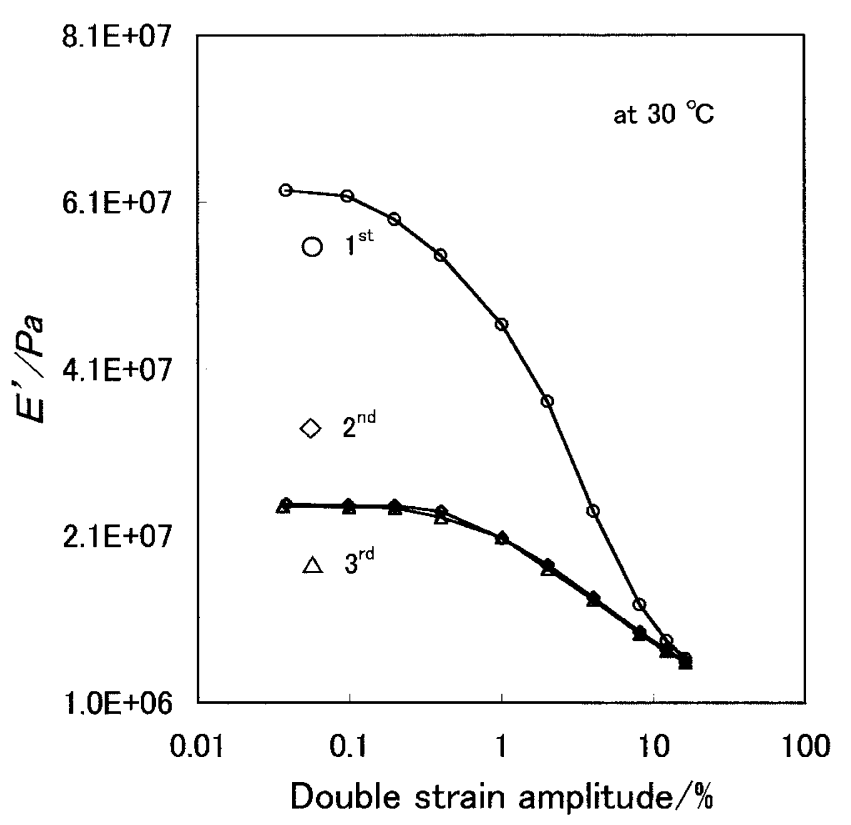

Figure 2. Strain dependence of storage modulus $E^{\prime}$ at $30^{\circ} \mathrm{C}$ and $11 \mathrm{~Hz}$ for annealed CPE/VAO-80 (30 wt\%).

ing point of the AO- 80 crystal.

Five domains can be identified on $E^{\prime}$ curve showed in Figure 1: two dispersion regions in which $E^{\prime}$ changes rapidly with temperature and three regions that show a much more gentle decrease in slope, resembling the behavior in the region of a plateau. What must be noted here is the value of $E^{\prime}$ in the intermediate plateau region. For pure $\mathrm{CPE}$, values of $E^{\prime}$ above the glass transition temperature are located in the range of $10^{6}-10^{7} \mathrm{~Pa}$. In contrast, annealed CPE/VAO-80 showed a very high $E^{\prime}$ value in the intermediate plateau region. This dramatic increase in $E^{\prime}$ in the intermediate plateau region suggests that that AO- 80 crystals within the CPE matrix act not only as a filler but also as multifunctional cross-links.

It is conceivable that a quite defective AO- 80 crystal formed within the CPE matrix during the annealing process is weak and that it may be destroyed under the condition of a relatively large deformation. Consequently, $E^{\prime}$ in the intermediate plateau region can be considered to be composed of the following two parts. One is independent of strain including the pure CPE network, the hydrodynamic effect of AO-80 crystal, specific interactions between AO-80 crystal and CPE matrix. Another is a strain-dependent part caused by AO-80 crystal network that tied with the incorporated $\mathrm{CPE}$ chains. If the latter were a case, the Payne effect as a measure of such a crystal network would be observed. In this study, we focused on the strain dependence of $E^{\prime}$ in the intermediate plateau region.

Figure 2 shows the strain dependencies of $E^{\prime}$ at $30^{\circ} \mathrm{C}$ and $11 \mathrm{~Hz}$ for annealed CPE/VAO-80 (30 wt \%). For 


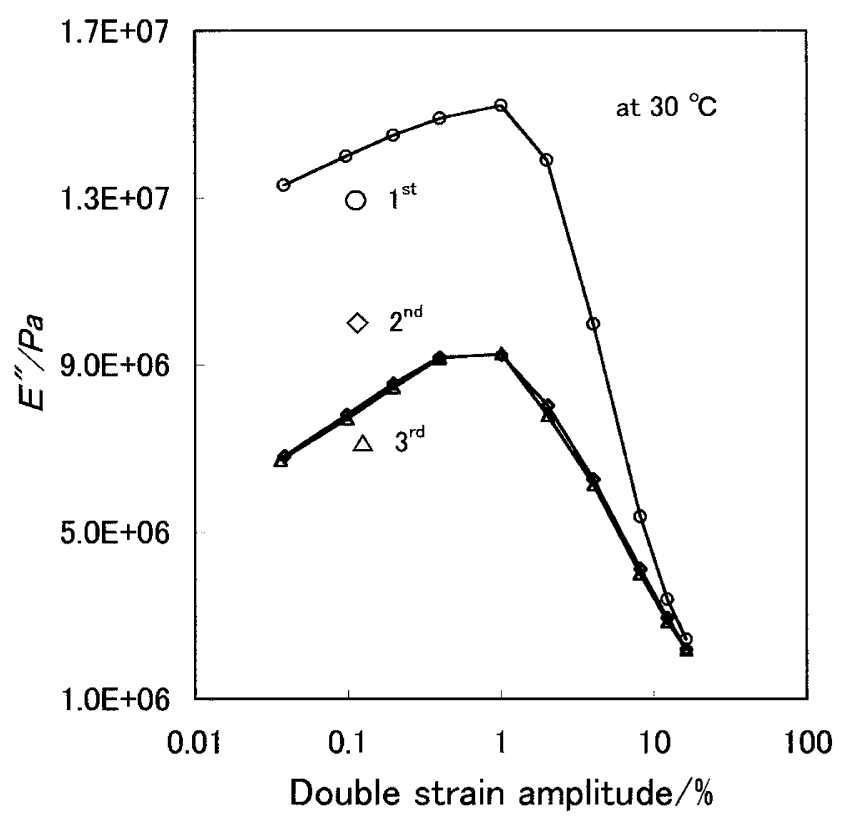

Figure 3. Strain dependence of loss modulus $E^{\prime \prime}$ at $30^{\circ} \mathrm{C}$ and $11 \mathrm{~Hz}$ for annealed CPE/VAO-80 (30 wt \%).

this sample, the effect of stain amplitude on $E^{\prime}$ is very pronounced. Thus, a temporary network of AO-80 crystals linked by the incorporated CPE chains was illustrated by the observed Payne effect.

The strain dependence of the dynamic tensile modulus of the annealed CPE/VAO-80 (30 wt $\%)$ that has undergone a large deformation is of crucial importance in characterizing the AO- 80 crystal network. The drop in $E^{\prime}$ over the range of stains investigated for the first measurement is ascribed to a decrease in the density of the cross-link points. This decrease is thought to be a consequence of the dissociation of these cross-link points under the condition of a large deformation. A comparison of the first and second measurements indicate that the strain dependence of $E^{\prime}$ of the second measurement is smaller. This decrease in $E^{\prime}$ implies destruction of a part of the AO- 80 crystal network was accompanied by a large dynamic deformation. The partial destruction of the network is most probably due to the dissociation of the intermolecular hydrogen bond between CPE and AO- 80 within the AO- 80 crystal that contained CPE chains. If hydrogen bonding is the main reason for the network formation, such temporary network should be recovered ether fully or partly by giving appropriate rest time. Assuming that once dissociated hydrogen bonds can be combined again, it is estimated that the height of the $E^{\prime}$ in the intermediate plateau increases with relaxation time. This has been demonstrated from a result shown in Figure 4 of ref 8. In contrast, the second and third measurements exhibited identical behavior. This implies that the network after the second measurement is reversible. To rationalize this finding, it is

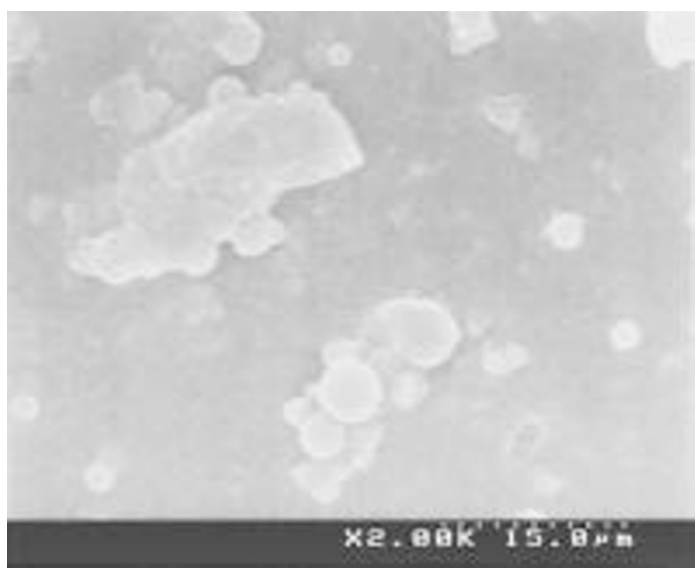

(a)

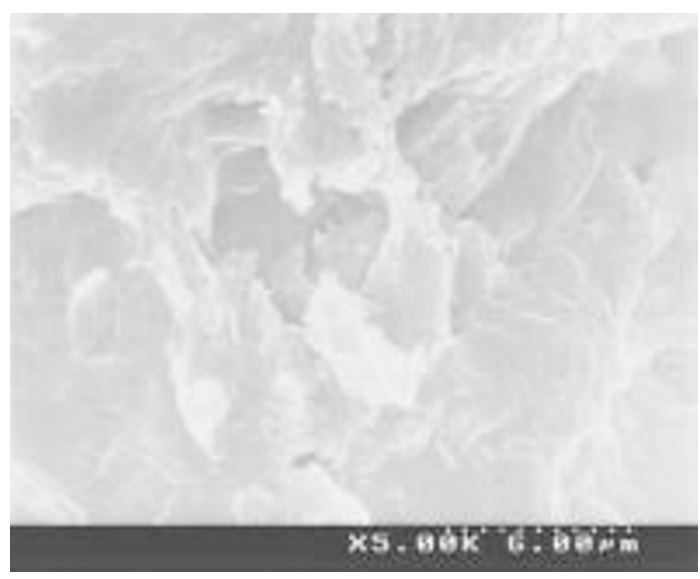

(b)

Figure 4. SEM photographs of untreated and annealed CPE/ VAO-80 (30 wt $\%)$.

essential to distinguish the intermolecular interaction in the AO-80 crystal that contained some CPE chains. In addition to the intermolecular hydrogen bond between CPE and AO-80, interactions between segments of one CPE chain and those of another CPE chain and/or an AO-80 molecule are also conceivable. Those interactions are probably due to van der Waals force and it can be broken when the strain (or stress) is sufficiently large. The decrease in $E^{\prime}$ at high strain amplitude for the second and third measurements is ascribed to the mechanical disruption of contact between segments of one CPE chain with those of another CPE chain and/or an AO-80 molecule. It should be stressed here that the repeated measurements of strain sweeps are a new method for estimating network strength.

In the case of filler-filled rubber, it is well known that the incorporation of filler in rubber will substantially increase the $E^{\prime \prime}$ of material regardless of the strain amplitude as observed with $E^{\prime}$, and this effect is primary attributed to the hydrodynamic effect as the addition of unstrained (or solid) particles in the polymer matrix would result in a high viscosity compound. Figure 3 shows the strain dependence of the $E^{\prime \prime}$ at $30^{\circ} \mathrm{C}$ and 
$11 \mathrm{~Hz}$. It was found that $E^{\prime \prime}$ shows a maximum value at a moderate strain amplitude (about $0.5 \%$ ). Such behavior cannot be explained only by the hydrodynamic effect since the $E^{\prime \prime}$ values of CPE while being lower, do not give significant strain dependence over a large practical range of strain amplitudes. It is also evident that, in contrast to $E^{\prime}$ of filled rubber which decreases monotonously with increasing strain, $E^{\prime \prime}$ shows a maximum values at a moderate strain amplitude, it is related to the breakdown and reformation of the structure. If the strain amplitude is small enough so that the network cannot be broken, $E^{\prime \prime}$ value seems to be determined mainly by the hydrodynamic effect of the AO- 80 crystal. On the other hand, if the strain is high enough that the network is destroyed to such an extent that it cannot be reconstructed in the time scale of dynamic frequency, the effect of the network on $E^{\prime \prime}$ will disappear. However, at a moderate strain, the network can be broken and then reconstructed rapidly, causing an additional energy loss. $E^{\prime \prime}$ is the sum of the hydrodynamic effect and the lost energy effect, and it therefore shows a maximum at a critical strain. On the other hand, such a maximum was not observed in the case of pure CPE. This also indicates the existence of an AO- 80 crystal network.

The results of the analyses of dynamic properties therefore suggest that AO-80 crystals in which CPE chains have been incorporated form a network. This suggestion was confirmed by SEM observations. Figure 4 shows SEM micrographs of two samples. In the case of an untreated sample, vitrified AO-80 particles were clearly observed as white agglomerates (> $10 \mu \mathrm{m})$ consisting of relatively small primary particles $(2-5 \mu \mathrm{m})$. In contrast, in the case of an annealed sample, only an irregular continuous phase could be seen, but a crystal ${ }^{9,10}$ such as that observed in the case of the CPE/AO-80 hybrid annealed at the same temperature did not appear. This indicates that the crystal of AO- 80 formed within the CPE matrix during annealing treatment is a mixed crystal and that such crystals are linked by incorporated CPE chains, forming a network.

\section{CONCLUSIONS}

The dynamic mechanical properties of an annealed
CPE/VAO-80 hybrid were investigated. The sample shows a new transition due to the melting of AO80 crystal formed in addition to the glass transition of CPE. Strain dependence of $E^{\prime}$ at temperatures below the melting point of AO-80 but above the glasstransition temperature was measured. The Payne effect, i.e., a decrease in $E^{\prime}$ with increase in strain amplitude and the appearance of a $E^{\prime \prime}$ maximum at strain of $0.5 \%$, was observed. This observation indicated the existence of a three-dimensional mobile physical network for the annealed CPE/VAO-80 hybrid. This network was confirmed by SEM observations. The Payne effect observed for the first measurement is composed of the following two parts: a part that is irreversible due to dissociation of intermolecular hydrogen bonds between CPE and AO-80 and another part that is reversible due to the slip of CPE chains within the AO- 80 crystal. After the second measurement, only the reversible part is observable. In addition, repeated measurements of strain sweeps has provided a new way for estimation of network strength.

\section{REFERENCES}

1. S. Vieweg, R. Unger, G. Heinrich, and E. Donth, Rubber Chem. Technol., 73, 495 (1999).

2. C. Wu, T. Yamagishi, Y. Nakamoto, S. Ishida, K. Nitta, and S. Kubota, J. Polym. Sci., Part B: Polym. Phys., 38, 2285 (2000).

3. C. Wu, S. Akiyama, T. Mabuchi, and K. Nitta, Polym. J., 33, 792 (2001).

4. C. Wu, Polymer, in press.

5. A. R. Payne, J. Polym. Sci., 6, 57 (1962).

6. A. R. Payne and R. E. Whittaker, Rubber Chem. Technol., 44, 1207 (1971).

7. M.-J. Wang, Rubber Chem. Technol., 71, 520 (1997).

8. C. Wu, J. Mater. Sci. Latt., 20, 1389 (2001).

9. C. Wu, T. Yamagishi, and Y. Nakamoto, Polym. Int., 50, 1095 (2001).

10. C. Wu and K. Nitta, Kobunshi Ronbunshu, 57, 449 (2000). 